Gopal R Sharma, MS

Department of Neurosurgery

National Academy of Medical sciences

Bir Hospital, Kathmandu, Nepal

Rajiv Jha, MS, M Ch

Department of Neurosurgery

National Academy of Medical sciences

Bir Hospital, Kathmandu, Nepal

Prakash Poudel, FCPS

Department of Neurosurgery

National Academy of Medical sciences

Bir Hospital, Kathmandu, Nepal

Dhrub R Adhikari, MS

Department of Neurosurgery

National Academy of Medical sciences

Bir Hospital, Kathmandu, Nepal

Prakash Bista, MS, M Ch

Department of Neurosurgery

National Academy of Medical sciences

Bir Hospital, Kathmandu, Nepal

Address for Correspondence:

Gopal R Sharma, MS

Department of Neurosurgery

National Academy of Medical sciences

Bir Hospital, Kathmandu, Nepal

Email: gopal47@hotmail.com

Received, 6 July, 2017

Accepted, 13 July, 2017

\section{Microvascular Decompression for Trigeminal Neuralgia: Our Experiences at Bir Hospital}

Trigeminal neuralgia (TGN) is a very peculiar disease, mostly characterized by unilateral paroxysmal facial pain, often described by patient as 'one of the worst pain in my life'. This condition is also known as 'Tic Douloureus'. The annual incidence of TN is about $4.7 / 100000$ population, male and female are equally affected. The diagnosis is usually made by history, clinical findings and cranial imaging is required to rule out compressing vascular loop, organic lesions and Multiple Sclerosis (MS) at Trigeminal nerve (TN). Treatment of TGN ranged from medical to surgical intervention.

Between September 2007 and April 2015, 20 patients underwent micro vascular decompression (MVD) of TN for TGN who were refractory to medical treatment at department of Neurosurgery, Bir Hospital. All decompressions were performed using operating microscope. Follow up period ranged from 22 months to 8 years.

There were 9 males and 11 females and age ranged from 30-70 years. The neuralgic pain was localized on right side in 13 patients and left on 7 patients. Pain distribution was on V3 (mandibular branch) dermatome in 11, V2( Maxillary branch ) in 4, V2-3 in 2 and V12-3 in 3 patients respectively. On intraoperative findings TN was compressed by superior cerebellar artery ( SCA ) in 8 , tumors in 4 , unidentified vessels in 3 , veins in 2, anterior inferior cerebellar artery ( AICA ) in 1 and no cause was found in 2 patients. 7 patients suffered postoperative complications which included hyposthesia in 3, pseudomeningocele in 3 and meningitis in 1 . There was no mortality in this series. 20 patients felt pain relief immediately after procedure and 1 patients came after 3 years with recurrent pain requiring second surgery.

In conclusion, MVD for TGN in younger patients who are refractory to medical treatment is one of the best treatment options which is safe and long term pain relief is achieved in majority of cases.

Key Words: microvascular decompression, recurrence, surgical results, trigeminal nerve, trigeminal neuralgia 


\section{Sharma et al}

Trigeminal neuralgia (TGN) is a clinical condition characterized by sudden sharp, shooting, lancinating pain lasting several seconds to minutes and localized to one, two or three branches of trigeminal nerves (TN). The pain is usually unilateral, however, bilateral facial pain have been reported in the literature. The attack of pain usually starts by stimulation of so called trigger zones like teeth brushing, eating, chewing, drinking, washing face etc. Annual incidence of TGN is $4.7 / 100,000$ population per year. $^{9}$

Most cases of TGN are still referred to as idiopathic, although many are associated with vascular compression of the trigeminal nerve. In minority of cases pain might be due to multiple sclerosis (MS) or nerve compression by tumors. ${ }^{16}$ TGN due to compression by tumors is about $4 \%$ and most common tumors are trigeminal schwannomas, acoustic neuromas, dermoid and epidermoids. Two percent $(2 \%)$ of patients with MS have TGN, where as $18 \%$ of patients with bilateral TGN have MS. ${ }^{13}$

Diagnosis is usually made by classical history, however, cranial imaging like CT Scan /MRI is indicated to exclude other pathology such as tumors, vascular malformation, MS and other abnormalities of brain Stem.

First line treatment of TN is always drug therapy and carbamazepine is the first choice of drug. Other drugs which are being used alone or in combination of carbamazepine are amitryptilline, gabapentine, pregabapentin and baclofen. $^{16}$

If medical treatment fails or is not tolerated surgical interventions are available. Surgical interventions ranged from ablative therapy like percutaneous rhizotomy to MVD. ${ }^{2,9}$ After the popularization of MVD by Peter Jannetta in 1970s, MVD became one of the best and widely used treatment option for TGN. ${ }^{8}$ Non invasive treatment like Gamma Knife/Cyber Knife has been shown effective treatment to relieve neuralgic pain in some patients, however, long term results are not available. ${ }^{4,10}$

In this study 20 patients suffering from TGN and underwent MVD were retrospectively analyzed and results were discussed with review of pertinent literature.

\section{Materials and Methods}

This is a retrospective study of case series of TGN who were not responding to medical treatment and underwent MVD after clinical and radiological evaluation. Details of cases were retrieved from admission charts, OT register, discharge summary files and follow up records. Between September 2007 and April 2015, 20 patients underwent MVD at department of Neurosurgery, Bir Hospital. All
MVDs were performed using operating microscope. We do not use intraoperative brainstem auditory evoked potentials monitoring. Follow up period ranged from 22 months to 8 years.

\section{Surgical Technique of MVD}

After general anaesthesia patient is kept in supine obligue or lateral decubitus or park bench position with head fixed to Mayfield's three pins after head is mildly flexed, rotated up to $10^{\circ}$ and making the head parallel to floor providing maximum exposure of postauricular retromastoid area. About $4-6 \mathrm{~cm}$ mildly curvilinear or straight skin incision is made just $1.5-2 \mathrm{~cm}$ behind or medial to the mastoid groove or notch. The extension of incision should be 1/3 above and 2/3 below the transverse sinus which should coincide with an imaginary transverse line drawn from tragus. Retromastoid suboocipital bone is exposed after deattachment of muscles and pericranium. A standard $2-3 \mathrm{~cm}$ diameter suboccipital craniectomy or craniotomy is performed at the junction of transverse and sigmoid sinuses and exposing the edges of transverse sinus above and sigmoid sinus anteriorly. The curvilinear dural opening is made and reflecting dural edges toward sigmoid and transverse sinuses. The cerebellar hemisphere is gradually retracted medially and CSF is drain and in many occasion cervicomedullary cistern needs to be punctured to drain CSF adequately to lax brain. At this point operating microscope is brought to the operating field. Now inside the CP angle area we can see petrosal vein, $7^{\text {th }} \& 8^{\text {th }}$ nerves and lower cranial nerves from above to downward. Arachnoid membrane covering the petrosal vein to 7 and 8 cranial nerves are opened up by sharp dissection. If petrosal vein is under tension or started bleeding, it is diathermized and divided. After opening arachnoid layer $5^{\text {th }}$ nerve is visualized which is located anterior to petrosal vein and anteriosuperior to $7^{\text {th }}$ and $8^{\text {th }}$ nerves.In majority of cases the offending vessel compressing dorsal REZ of TN is found. Occasionally, more than one vessel may be identified. The vascular loop is well recognized and displaced with sharp dissecton and a Teflon or muscle piece is placed in between TN and vascular loop to avoid contact. After proper hemostasis dura is closed in water tight fashion. The wound closed in anatomical layers after replacing and repositioning bone flap if free bone flap has been raised previously.

\section{Results}

Twenty patients underwent MVD for TGN who were refractory to medical treatment. There were 9 male and 11 female and age ranged from 30- 70 years. Pain was localized to right face in 13 patients and to the left in 7 patients. Branches involved were V3 (11), V2 (4), V2 \& 
V3 (2) and V1 V2 \& V3 (3) respectively (Table 1). All 20 patients undergone MVD had intractable facial pain despite multi drugs therapy treatment. Prior to surgery, all patients were advocated for cranial MRI. 16 patients had normal cranial imaging and 4 patients had cerebellopontine angle masses. All patients underwent standard unilateral suboccipital retromastoid craniectomy and MVD of TGN in park bench position under general anaesthesia. MVD was performed using operating microscope in all cases.TN were compressed by SCA in 8 , tumors in 4, unidentified vessels in 3, veins in 2, AICA in 1 and no cause was found in 2 (Table 2). 7 patients suffered postoperative complications which included hyposthesia in 3, pseudomeningocele in 3 and meningitis in 1 patient (Table 3). There was no surgery related mortality in our series. Pain was relieved immediately after MVD in all cases. All patients were discharged from hospital within 7 to 10 days after postoperative cranial imaging. There was one patient who came after 3 years of MVD with recurrence of pain and underwent second decompression (Table 4).

\begin{tabular}{|l|l|}
\hline Trigeminal nerve branches & No of cases (\%) \\
\hline V2 & $4(20 \%)$ \\
\hline V3 & $11(55 \%)$ \\
\hline V2 \& V3 & $2(10 \%)$ \\
\hline All & $3(15 \%)$ \\
\hline
\end{tabular}

Table 1: Pain distribution

\begin{tabular}{|l|l|}
\hline Causes of compression & No of patients (\%) \\
\hline Superior cerebellar artery (SCA) & $8(40 \%)$ \\
\hline $\begin{array}{l}\text { Anterior inferior cerebellar artery } \\
\text { (AICA ) }\end{array}$ & $1(5 \%)$ \\
\hline Veins & $2(10 \%)$ \\
\hline Unidentified vessels & $3(15 \%)$ \\
\hline CP angle mass & $4(20 \%)$ \\
\hline No cause & $2(10 \%)$ \\
\hline
\end{tabular}

Table 2: Etiology of trigeminal neuralgia

\begin{tabular}{|l|l|}
\hline Complications & No of cases (\%) \\
\hline Facial hyposthesia & $3(15 \%)$ \\
\hline Pseudomeningocele & $3(15 \%)$ \\
\hline Meningitis & $1(5 \%)$ \\
\hline
\end{tabular}

Table 3: Post operative complication of MVD

\begin{tabular}{|l|l|l|}
\hline Outcome & No. of cases & Percentage (\%) \\
\hline Immediate pain relief & 20 & 100 \\
\hline Long term pain free & 19 & 95 \\
\hline Recurrence & 1 & 5 \\
\hline Morbidity & 7 & 35 \\
\hline Mortality & 0 & 0 \\
\hline
\end{tabular}

Table 4: Outcome of MVD

\section{Discussions}

If a patient does not obtain relief from neuralgic pain with medical treatment, then some form of interventions may be advocated. It is estimated that up to $50 \%$ of the patients suffering from TGN will sooner or later be in that situation ${ }^{4}$. Among invasive procedures, there are mainly two, one is ablative and other one is non destructive procedure. Ablative procedures are lesioning nerve fibers or ganglion cells such as peripheral nerve section, ganglionectomy, rhizotomy etc. Percutaneous procedures like Chemical rhizotomy( Glycerol ), Thermal coagulation, balloon compression of Gasserian ganglion are commonly practiced ablative procedures which are comparatively safe and less invasive. ${ }^{12}$

More recently Gamma Knife therapy has been used to make a lesion in the trigeminal dorsal REZ by means of a stereotactic technique, however, its efficacy and long term results are not available. ${ }^{4,10}$

The non destructive procedure is MVD, which is widely accepted main surgical option for TGN these days.

Walter Dandy used to do open trigeminal nerve sensory fibers section for TGN and in many occasions during surgery he found vascular loops compressing the trigeminal nerve. Then in 1932, he proposed that trigeminal neuralgia could be due to a mechanical compression or irritation of the nerve root. ${ }^{4}$ In 1959, James Gardener tried to perform MVD but he could not succeed due to lack of proper instruments. ${ }^{7}$ On this basis, Peter Jannetta, in 1967, performed first successful MVD using operating microscope and microinstruments and later on he popularized it. ${ }^{8}$ Now a days, MVD became a widely applied procedure for TGN.

Patients who are refractory to medical treatment and/ or who developed drug intolerance like allergic reaction, are the ideal candidates for MVD. Predicting outcome of MVD for individual patients is difficult, however, previous experiences have shown that if the patient is male, has immediate postoperative pain relief, if there is no cause of venous compression, if shorter duration of disease and no previous surgery, respond better to MVD and offers best 
long term cure rates..$^{1,5,11,13,15}$ Outcome also varies with the case load of the operating surgeons.

MVD is a non destructive procedure and curative in more than $85 \%$ of cases, however, it is a major surgical undertaking and should be performed under general anaesthesia, so best avoided in old age with comorbidity. Though rare, lethal complication like meningitis can occur which may kills patient if not diagnosed and treated in time. ${ }^{5,11}$ The general morbidity such as hearing loss and sensory disorders in trigeminal distribution varies between 0.2 and $4.5 \% .{ }^{13,15}$ The aim of operation is to remove the suspected compression of the nerve by a loop of an artery or sometimes a vein near brainstem at dorsal REZ. Gold standard procedure is microscope assisted MVD which has been practicing widely by neurosurgeons, however, since last few years endoscope assisted MVD is gradually emerging procedure but its long term results are not available. ${ }^{3}$

Most common compressing structures found during MVD are SCA, AICA, arteries, veins and tumors. In Jannetta's series of 163 cases, common compressing structures were SCA ( 82 ), AICA ( 9 ), veins ( 19 ), arteries and veins ( 22 ), tumors ( 13 ), multiple sclerosis ( 4 ), atrophic nerve ( 2 ). ${ }^{11}$ In Oseman series of 156 patients, TN was compressed by SCA in $66 \%, 14 \%$ by arterial and venous compression, $9 \%$ by only veins, $3 \%$ by tumors and in $6 \%$ there was no compression. ${ }^{13}$ In Bohman and et al's series of 47 patients, SCA was the most common cause of TN compression in $89 \%$ of patients. ${ }^{3}$ Similarly in our present series Compression of TN was caused by SCA in 8 patients, tumors in 4 , unidentified vessels in 3, veins in 2, AICA in 1 and no cause was found in 2 .

One of the most common complication after MVD is hyposthesia over the trigeminal dermatome and 3 patients in our series had developed hyposthesia which was transient. 11 out of 362 patients in a series of Marc Sindou, had developed postoperative hyposthesia, paresthesia and most of them were permanent deficit ${ }^{15}$. In Oesman's series of 156 cases, $13 \%$ patients had suffered hyperpathia and hyposthesia. Other cranial nerve deficits like 7, 8, 9, 10 and 11 were rare. ${ }^{13}$ Transient complications like dizziness, nausea or headache are othercommon complications. ${ }^{1,2,9,11,13}$ MVD seems to be a safe procedure, however, occasionally CSF leak and meningitis can occur. ${ }^{13,15}$ In our series one patient had developed meningitis after CSF leakage from the wound which required wound repair, lumbar drain for one week and IV antibiotics for two weeks.

Surgery related death is remote in MVD for TN. There was no mortality in Oesman's series of 156 cases. ${ }^{13}$ There was no surgery related mortality in Marc Sindou's series of 362 cases as well. ${ }^{15}$ In Peter Jannetta's series of 1185 cases there was $0.2 \%$ mortality. ${ }^{11}$ In our series there was no surgery related death.

As mentioned earlier long term pain relief after MVD rely on etiology, type of TGN, age of the patients and duration of symptoms before surgical intervention. $1,5,8,11,13,15$ Long term cure of neuralgia can be achieved in more than $80 \%$ of cases. ${ }^{5,13,11,15}$ In Marc Sindou's series immediate pain relief was achieved in $85 \%$ and long term pain relief was in $73.38 \%{ }^{15}$ In Oesman's series $88 \%$ reported immediate pain relief and $82 \%$ had long term pain relief. ${ }^{13}$ We have similar experienes of having $100 \%$ immediate pain relief and $95 \%$ in 22 months to 7 years followed up period.

Recurrence of pain after MVD is one of the common clinical scenario. Recurrence is about $20-30 \%$ in most of the published series of MVD. ${ }^{1,2,13,14}$ In Barker and Jannetta's series of 1185 cases recurrence rate was 30\% in 20 years followed up period and most of the recurrence occurred within first two years after surgery. ${ }^{1}$ Burchiel et al reported $6 \%$ recurrence at 60 months and $42 \%$ in 100 months. ${ }^{5}$ In our series, there was $5 \%$ recurrence in 3 years' period which required second procedure.

Conclusion: MVD for TN in younger patients who are refractory to medical treatment is one of the best treatment options which is safe and long term pain relief is achieved in majority of cases.

\section{References}

1. Joffroy A, Levier M, Massager N. Trigeminal neuralgia: pathophyiology and management. Acta Neurol belg 101: 20-25,2001

2. Bennetto L, Patel NK, Fuller G. Trigeminal neuralgia and its management. BMJ 334: 201-205, 2007

3. Oesman C, Mooij JJA. Long term follow up of microvascular decompression for trigeminal neuralgia. Skull Base 21(5): 313-322, 2011

4. Talor JC. Long term treatment of trigeminal neuralgia with carbamazepine. Postgrad Med J 57: 16-18, 1981

5. Jannetta PJ. Arterial compression of trigeminal nerve at pons in patients with trigeminal neuralgia. $\mathbf{J}$ Neurosurg 26 (1): 159-162, 1967

6. Brisman R. Gamma Knife radiosurgery for primary management for trigeminal neuralgia. J Neurosurg (Suppl 3) 93: 159-161,2000

7. Linskey M, Ratanatharathorn V, Penagaricano J. A prospective cohort study of microvascular decompression and Gamma Knife surgery in patients with trigeminal neuralgia. J Neurosurg 109: 160- 
172,2008

8. Nurmikko TJ, Eldridge PR. Trigeminal neuralgiapathophysiology, diagnosis and current treatment. Br J Anaesth 87 (1): 117-132, 2001

9. Dandy WE. Concerning the cause of trigeminal neuralgia. Am J Surg 24: 44-455, 1934

10. Gardner WJ, Miklos MV. Response of trigeminal neuralgia to "decompression" of sensory root. J Am Ass 170: 1773-76, 1959

11. Lovely TJ, Jannetta PJ. Microvascular decompression for trigeminal neuralgia. Surgical technique and long term results. Neurosurg Clin N Am 8(1): 1129,1997

12. Baker FG, Jannetta P, Bissonette DJ, Larkin MV, Jho HD. The long term outcome of microvascular decompression for trigeminal neuralgia. N Engl Med 334: 1077-1084,1996

13. Burchiel KJ, Clarke H, Haglend M, Loeser J. Long term efficacy of microvascular decompression in trigeminal neuralgia. J Neurosurg 69: 35-38, 1988

14. Bohman LE, Pierce J, Stephen JH, Sandhu S, Lee JYK. Fully endoscopic microvascular decompression for trigeminal neuralgia: technique review and early outcomes. Neurosurg Focus 37(4); 18, 2014

15. Sindau M, Leston J, Decullier E, Chapuis F. Micrvascular decompression for primary trigeminal neuralgia: Long term effectiveness and prognostic factors in a series of 362 consecutive patients with clear cut neurovascular conflicts who underwent pure decompression. J Neurosurg 107; 1144-1153, 2007

16. Sanchez - Meha RO, Limbo M, Cheng JS, Camara J, Ward MM, Barbaro NM. Recurrent or refractory trigeminal neuralgia after microvascular decompression, radiofrequency ablation, or radiosurgery. Neurosurg Focus 18 (5): E12, 2005 